Philosophische Masken 


\section{Philosophische Masken}

Literarische Formen der Philosophie bei Platon, Descartes, Wolff und Lichtenberg 


\section{CIP-Titelaufnahme der Deutschen Bibliothek}

Schildknecht, Christiane:

Philosophische Masken : literarische Formen der Philosophie

bei Platon, Descartes, Wolff und Lichtenberg / Christiane

Schildknecht. - Stuttgart : Metzler, 1990

ISBN 978-3-476-00717-9

ISBN 978-3-476-00717-9

ISBN 978-3-476-03326-0 (eBook)

DOI 10.1007/978-3-476-03326-0

Dieses Werk einschließlich aller seiner Teile ist urheberrechtlich geschützt. Jede Verwertung außerhalb der engen Grenzen des Urheberrechtsgesetzes ist ohne Zustimmung des Verlages unzulässig und strafbar. Das gilt insbesondere für Vervielfältigungen, Übersetzungen, Mikroverfilmungen und die Einspeicherung und Verarbeitung in elektronischen

Systemen.

(C) 1990 Springer-Verlag GmbH Deutschland

Ursprünglich erschienen bei J.B. Metzlersche Verlagsbuchhandlung und Carl Ernst Poeschel Verlag GmbH in Stuttgart 1990 
meinen Eltern 
INHALT

Vorbemerkung 11

Einleitung 13

I. Platon oder die dialogische Form der Philosophie 22

1. Der Platonische Begriff philosophischen Wissens: „Richtige Vorstellung und Erkenntnis sind etwas Verschiedenes* 23

1.1. Formen praktischen Wissens - Von Augenzeugen und Wegekundigen, von Weberschiffchen, Zaumzeug und Flöte 26

1.2. Formen intuitiven Wissens: "Über dem Himmel, die Sterne betrachtend 30

1.3. Dialektisches Wissen - Eine gehörige Seele wählen und mit Einsicht Reden säen und pflanzen 34

2. Die Darstellungsform des Dialogs: ^In der Philosophie leben* 38

2.1. Ungleiches Wissen: „Du wirst nur, lieber Glaukon, nicht mehr imstande sein zu folgen!* 38

2.2. Die Platonische Schriftkritik - Aussparung und »leiser Fingerzeig* 43

II. Descartes oder die monologische Form der Philosophie 54

1. Formen der Subjektivität - Gründe, »die nur unserem Denken entnommen sind « 57

1.1. Monologische Wissensbildung: „Einsam und zurückgezogen leben wie in der entlegensten Wildnis* 57

1.2. Dialogische Wissensbildung - Der "Stil der Unterhaltung* gegen die Trockenheit scholastischer Philosophie 64

2. Die Cartesische Methode - Wissensbildung waus eigener Erkenntniskraft* 68

3. Intuition und Illumination - Die Funken des Wissens 70

4. Selbststilisierung und Tarnung: "Larvatus prodeo* 75 
1. Die selbstreflexiv-apologetische Komponente - Vita, fata et scripta 85

2. Wolffs Begriff philosophischen Wissens und die Form des Lehrbuchs Der "Geist der Gründlichkeit* 92

2.1. Die axiomatische Methode: Der "Plan des Mathematikers im Kopfe* oder die *richtig bewiesene Weltweisheit 92

2.2. Die Lehrbuch-Philosophie 103

2.2.1. Die deutschen und lateinischen Lehrbücher: "Meine Worte fallen, wie ich denke* 103

2.3. Grenzen des propositionalen Wissens bei Wolff - Die "gar seichte Art zu philosophieren* 109

IV. Lichtenberg oder die aphoristische Form der Philosophie 123

1. Lichtenbergs Wissensbegriff 124

1.1. Der Experimentalphysiker: „Man muß etwas Neues machen, um etwas Neues zu sehen* 124

1.2. Der Experimentalphilosoph: „Die Frage: soll man selbst philosophieren? muß dünkt mich so beantwortet werden als eine ähnliche: soll man sich selbst rasieren* 137

2. Komponenten der Nicht-Propositionalität:

"Himmel gefühlt ist ausgedruckt Nonsense Nonsense* 148

3. Die Form der Darstellung: "Wie es denn wirklich an dem ist, daß Philosophie, wenn sie für den Menschen etwas mehr sein soll als eine Sammlung von Materien zum Disputieren, nur indirekte gelehrt werden kann* 157

Schlu 170

Literaturoerzeichnis 173

Personen- und Sachregister 186 
The whole man must move together.

Die Vergehungen dagegen sind unzählbar, und der Schaden der daraus entsteht groß und öfters unersetzlich. Zum Menschen rechne ich Kopf Herz Mund und Hände, es ist eine Meister-Kunst diese durch Wind und Wetter unzertrennt bis an das Ende zu treiben, wo alle Bewegung aufhört.

(Lichtenberg)

Ich kann freilich nicht sagen, ob es besser werden wird, wenn es anders wird; aber so viel kann ich sagen, es muß anders werden, wenn es gut werden soll.

(Lichtenberg) 


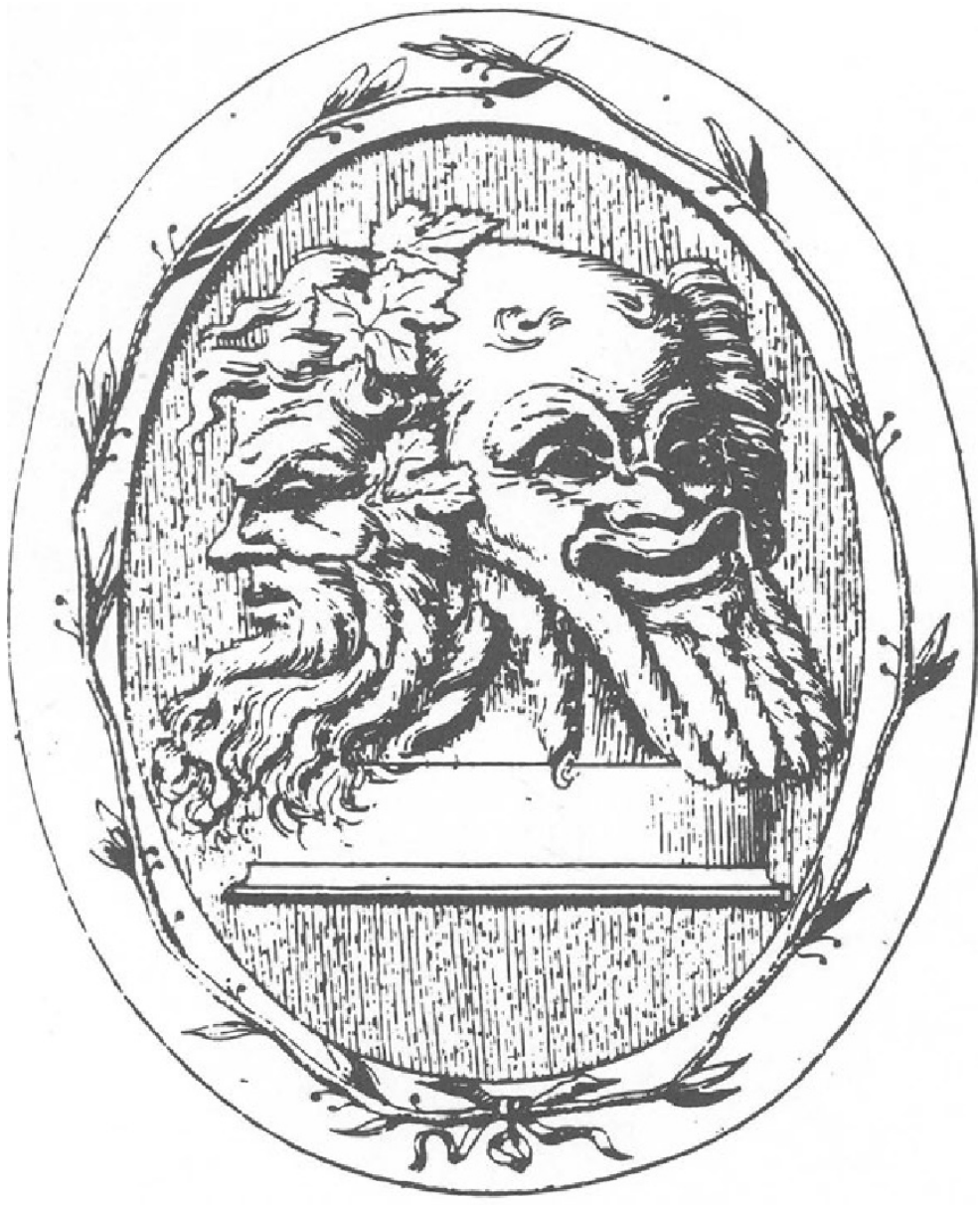




\section{VORBEMERKUNG}

Die Stellung der Philosophie zwischen Dichtung und Wissenschaft spiegelt sich in dem breiten Spektrum ihrer literarischen Darstellungsformen. Diese geben denn auch Anlaß zu Betrachtungen der unterschiedlichsten Art. Die vorliegende Arbeit, die eine leicht überarbeitete Version meiner Dissertation (Universität Konstanz, Sommer 1989) darstellt, nähert sich dem Thema aus erkenntnistheoretischer Sicht: Welcher Begriff philosophischen Wissens liegt jeweils zugrunde und in welcher Beziehung steht er zu der gewählten Form der Darstellung dieses Wissens? Anhand von vier Philosophen - Platon, Descartes, Wolff und Lichtenberg - wird diese Frage exemplarisch im Hinblick auf eine methodische Bedeutung literarischer Formen in der Philosophie beantwortet und damit eine Alternative zu szientistischen und dekonstruktivistischen Ansätzen aufgezeigt.

Das Kapitel zur literarischen Form bei Descartes ist in einer leicht veränderten Fassung bereits in: Gottfried Gabriel/Christiane Schildknecht (eds.), Literarische Formen der Philosophie, Stuttgart 1990, 92-120 erschienen. Dieser Sammelband widmet sich der obengenannten Fragestellung unter erweiterter Autorenund Formperspektive und enthält in einer Bibliographie weiterführende Literatur zu diesem Thema.

Für vielfältige Formen der Unterstützung und Betreuung - aufmunternde Worte und emphatisch geführte Diskussionen auch über weite Distanzen möchte ich zuerst den Konstanzer Professoren Jürgen Mittelstraß und Gottfried Gabriel danken. Wertvolle Kritik und rettende Hinweise zu einzelnen Kapiteln verdanke ich besonders Dr. Burkhart Steinwachs, Prof. Thomas A. Szlezák, Prof. Catherine Wilson und Prof. Gereon Wolters. Der Studienstiftung des deutschen Volkes gilt mein Dank für die Gewährung eines Promotionsstipendiums. Mit in Worten nicht aufwiegbarer Wärme und Geduld haben mir Ulrike Borst, Soraya de Chadarevian, Wolfgang Ferchl, Artur Göser, Iris Hentschel, Christiane und Peter Jakob-Lanz, Angela Mössle, Peter Ohlendorf und Frauke Riller geholfen, die gerufenen Promotionsgeister schließlich auch wieder loszuwerden. 